\title{
Continuous Gulture of Torulopsis utilis: A Kinetic Study of Oxygen Limited Growth
}

\author{
BY D. K. BUTTON* AND J. C. GARVER \\ Department of Biochemistry, University of Wisconsin, \\ Madison, Wisconsin, U.S.A.
}

(Received 29 November 1965)

\begin{abstract}
SUMMARY
A small single-phase continuous fermentor system for convenient longterm operation at steady state with small population densities is described. A method by which the effect of extracellular limiting nutrient on growth rate can be evaluated from the resulting data is discussed. This method was used for Torulopsis utilis to determine the Michaelis constants for growth with oxygen and glycerol; these were found to be $1 \cdot 4 \times 10^{-5} \mathrm{M}$ and $4.9 \times 10^{-5} \mathrm{M}$. Size of organisms and yields were found to decrease with growth rate. Average volume of organisms decreased from 60 to $41 \mu^{3}$ and the yield decreased from 1.8 to $1.2 \mathrm{~g}$. dry wt./organism/g. oxygen consumed. An endogenous metabolism rate constant determined from yields at growth rates above $30 \%$ maximum was 0.056 g. oxygen used/g. dry wt. organism/ hr.
\end{abstract}

\section{INTRODUCTION}

Among the advantages of continuous cultures of micro-organisms is the production of a culture grown at a fixed rate by the controlled extracellular concentration of a single nutrient. The fact that growth rate is determined by a limiting nutrient concentration and that population density is fixed by the amount of limiting nutrient absorbed can be utilized to relate growth to limiting substrate concentration. This relationship was shown to fit the well-known Michaelis-Menten equation by Monod (1942) where the Michaelis constant $K_{s}$ was defined as the concentration of limiting substrate at which microbial growth velocity was half maximum. Monod's method of determining $K_{s}$ was to measure growth rates in batch culture at known initial sugar concentrations. Herbert, Elsworth, \& Telling (1956), in an extension of the original continuous culture theory presented by Monod (1950), attempted to measure $\boldsymbol{K}_{s}$ for glycerol by analysis of the effluent of a glycerol-limited continuous culture. Davies, Karush \& Rudd (1965) used the same technique to analyse fermentor effluents for extracellular and limiting quantities of radioactive amino acids. It was recognized by Rao (1962) that decreasing steady-state populations at decreasing substrate concentrations in the feed of a continuous culture predict, when extrapolated to zero population size, the substrate concentration which surrounds the organisms at a particular growth rate. Rates of oxygen disappearance in static culture have been measured polarographically by Longmuir (1954) at various oxygen concentrations. The purpose of the present work is to

\footnotetext{
* Present address : Institute of Marine Science, University of Alaska, College, Alaska, 99735.
} 
relate microbial growth rates to dissolved oxygen concentration in the medium under conditions of oxygen limited growth.

Theory. A continuous culture is a continuous flow stirred tank reactor of the type described by Danckwerts (1954) adapted to grow micro-organisms. The autocatalytic growth reaction therein utilizes part of the limiting nutrient and the remainder is removed with the reaction product organisms. In a completely mixed reactor, the most efficient for autocatalytic processes (Herbert et al. 1956), this effluent substrate concentration is the same as the nutrient concentration which surrounds the organisms. The growth velocity of a culture limited by the concentration of a single limiting nutrient proceeds as a monotonic increasing function of substrate concentration (Monod, 1942) and the population of organisms proceeds directly to an equilibrium steady-state concentration (Button, 1964) fixed by the concentration and the rate at which substrate is supplied to the reactor. The above is widely recognized and can be formulated mathematically as follows:

Rate of change of concentration of organism = growth rate-output rate, i.e.

$$
\mathrm{V}(d X / d \theta)=\mu X V-F X,
$$

where $V$ is the volume of the reactor, $X$ the cell population, $\mu$ the specific growth rate of the organisms $(1 / X)(d X / d \theta), F$ the feed rate of fresh medium and $\theta$ time. At steady state, the concentration of organisms is constant with respect to time, $d X / d \theta=0$ and

$$
\mu X V=F X \text {. }
$$

Designating the specific through-put rate as $F / V$ and dividing through by $V$ gives :

$$
\mu=r \text {. }
$$

Thus at steady state the growth rate is equal to the dilution rate and can be set at any desired value between zero and the maximum growth rate of the organism, $\mu_{\text {max }}$, by suitable adjustment of the feed rate $F$.

The limiting nutrient balance in reactor. Rate of substrate concentration change = input rate-output rate - rate of substrate utilization for growth; i.e.

$$
V(d S / d \theta)=F S_{0}-F S-\mu(X / \gamma) V,
$$

where $S_{0}$ and $S$ are the feed and reactor limiting nutrient concentrations, respectively, and $\gamma$ is a yield constant relating dry weight of organisms produced to mass of limiting substrate utilized, or $X /\left(S_{0}-S\right)$. Then at steady state where $X$ and $S$ are at equilibrium values and $d S / d \theta=0$,

$$
F\left(S_{0}-S\right)=\mu(X / \gamma)
$$

since $r=F / V$ and $\mu=r$, one can solve for $S$ to give concentration of limiting nutrient in the reactor surrounding the organisms:

$$
S=S_{0}-(X / \gamma)
$$

The value of $S$ can be determined at any given growth rate by measuring the population density $X$ at a series of feed substrate concentrations $S$ and extrapolating to zero population size according to the equation

$$
X=\gamma\left(S_{0}-S\right)
$$


Since the growth rate $\mu$ can be varied by changing the dilution rate of the system according to equation (3), the growth rate can be related to the limiting nutrient concentration by determining $S$ as above at a series of dilution rates. The yield constant $\gamma$ has no effect on the extrapolation to $S$, hence different yields at different growth rates do not affect this method of substrate analysis. The only assumptions made in deriving the above equations are: (1) The growth rate is a function only of the concentration of a single limiting substrate, $\mu=f(S)$. (2) A range of steadystate stable conditions can be maintained in the fermentor. This implies that the net behaviour of the cells does not change with respect to time under steady-state conditions. (3) Perfect mixing exists in the fermentor. (4) All the organisms produced are viable.

\section{METHODS}

Extracellular nutrient concentration determination by the above method improves with operation of smaller population densities, where the extrapolation to zero population density is short. At these low population densities, a non-homogeneous distribution of organisms (part of the organisms growing on the fermentor

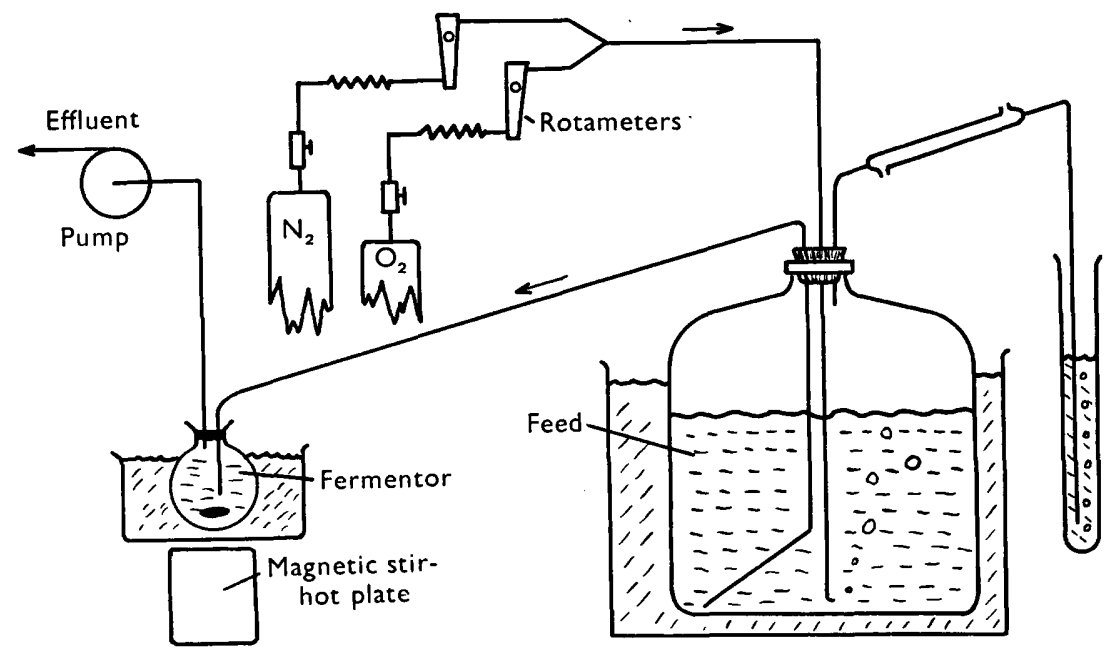

Fig. 1. Small single phase continuous fermentor.

walls, for example) render this method of nutrient concentration determination useless. A continuous culture system was thus designed specifically for operation at small population densities with special attention directed toward maintaining a uniform population as well as accurate regulation of the culture volume, feed rate, and limiting substrate concentration.

Apparatus. The continuous culture apparatus used consisted of a $40 \mathrm{l}$. Pyrex feed carboy, a $500 \mathrm{ml}$. round bottom flask reactor and a pump connected as shown in Fig. 1. Oxygen was supplied to the fermentor as one of the components dissolved in the feed. The fermentor was completely filled, thus eliminating a gas phase and associated problems in the growth portion of the system.

Feed-dissolved oxygen content was regulated by sparging the desired oxygen + nitrogen + dry air ratio into the feed carboy. Higher oxygen concentrations were 
obtained by mixing streams of the pure gases at fixed flow rates. These flow rates were regulated by controlling the pressure above a resistance bed of $300 \mu$ diam. glass beads packed into a section of capillary tubing. The streams were fed through calibrated rotameters for measurement before joining. Gas pressure was decreased and controlled with a series of three diaphragm pressure regulators. Compressed gas cylinders of oxygen in nitrogen were purchased (with analysis; The Matheson Company, Inc., Joliet, Illinois, U.S.A.) for obtaining the lower oxygen concentrations. Effluent gas was discharged into a column of ethylene-glycol to provide positive hydrostatic pressure at the level of the pump.

The 40 l. carboy was mounted in a constant temperature bath, all of which could be detached from the rest of the system and autoclaved as a unit. Ports were provided, in a rubber-stopper which closed the carboy, for a thermometer well, feed siphon, substrate additions and gas sparging. Additions to the carboy were injected through a syringe needle piercing a rubber diaphragm which covered one of the ports.

Feed was pumped into the fermentor, a $500 \mathrm{ml}$. round-bottom flask, positioned in a constant temperature bath over a magnetic stirrer. A 2 in. stir bar which mixed the system was driven at about $600 \mathrm{rev} . / \mathrm{min}$.; it dissipated a small volume of injected dye in less than a second. Feed and fermentor temperatures were accurately controlled at $35^{\circ}$.

Flow rate through the system was metered with a positive displacement Milton Roy controlled volume pump. The pump discharged effluent culture through a section of thermometer capillary, thus providing back pressure to prevent siphoning through the system. This arrangement allowed feed rates to be set within a few parts per thousand. Large-bore capillary tubing joined with sections of rubber hose connected the various components. These joints were wrapped with Mylar tape to decrease oxygen diffusion into the system; however, this precaution turned out to be unnecessary.

Organism. Several months of effort failed to find a suitable medium or fermentor surface which would allow the use of Escherichia coli as a test organism because of its tendency to adhere to solid surfaces. Although not apparent to the eye for the first few days, inconsistencies in the data indicated that a film of organisms was forming on the fermentor wall; this could later be seen. Therefore a different organism, Torulopsis (Candida) utilis 3, was chosen on the basis of its larger size and rapid growth in simple media. This organism did not adhere to the reactor walls and handled well. Later Mr D. Weinshank of this laboratory found that Bacillus megaterium was suitable for work at small population densities. The marine yeast, Cryptococcus albidus, is free from sticking tendencies in continuous culture (D. K. Button, unpublished observations).

Medium. The medium used was designed to study the effect of extracellular dissolved oxygen on the growth rate of Torulopsis utilis at small population densities. Thus additional required nutrients were adjusted between limits of concentration large enough to insure lack of growth limitation and small enough to insure absence of inhibition over the range of population densities required. Available inorganic analyses of yeast data were used to suggest the proper ratio of these constituents.

The medium was designed to accommodate a population density equivalent to about 0.05 g. dry wt. organism/l. Trace metal concentration adjustments were made to give a minimum lag phase before exponential growth in batch fermentations at 


\section{Continuous culture of torulopsis}

small population densities. Equilibrium amounts of dissolved oxygen were varied from $0 \cdot 1$ to $23 \cdot 1 \mathrm{mg}$. $\mathrm{O}_{2} / \mathrm{l}$. medium as calculated according to Henry's law and gas solubilities in dilute salt solutions. Glycerol was used as a convenient carbon source to avoid anaerobic metabolism confusing the experiments. Figure 2 shows relative steady-state population densities at a fixed oxygen concentration in the feed medium over a range of glycerol concentrations. These data show that the population density when oxygen was limiting was independent of concentration of carbon source. Since the data were collected at half maximum growth rate, the population densities extrapolate to a Michaelis constant for glycerol of $3.5 \mathrm{mg} . / 1$. Other constituents of the medium were (per 1.): $\mathrm{NaH}_{2} \mathrm{PO}_{4} . \mathrm{H}_{2} \mathrm{O} 2$ g., $\left(\mathrm{NH}_{4}\right)_{2} \mathrm{SO}_{4} 325 \mathrm{mg}$., $\mathrm{KCl} 40 \mathrm{mg}$., $\mathrm{MgSO}_{4} 25 \mathrm{mg}$., $\mathrm{CaCl}_{2} \cdot 2 \mathrm{H}_{2} \mathrm{O} 5 \mathrm{mg}$., $\mathrm{ZnSO}_{4} \cdot 7 \mathrm{H}_{2} \mathrm{O} 0 \cdot 7 \mathrm{mg}, \mathrm{Fe}\left(\mathrm{HN}_{4}\right)_{2}\left(\mathrm{SO}_{4}\right)_{2} \cdot 6 \mathrm{H}_{2} \mathrm{O}$ $0.5 \mathrm{mg}$., $\mathrm{CuSO}_{4} .5 \mathrm{H}_{2} \mathrm{O} 25 \mu \mathrm{g}$., $\mathrm{MnSO}_{4} . \mathrm{H}_{2} \mathrm{O} 1.5 \mu \mathrm{g}$., $\mathrm{CoSO}_{4} .7 \mathrm{H}_{2} \mathrm{O} 1.5 \mu \mathrm{g}$., $\mathrm{MoO}_{3}$ $0.5 \mu \mathrm{g}$., biotin $1 \mu \mathrm{g}$. Growth rate was independent of $\mathrm{pH}$ value between 4 and 7 . The medium was buffered at the lower value to decrease the probability of successful contamination.

Operational procedure. The medium was prepared in $40 \mathrm{l}$. carboys with distilled water, filtered through $0.45 \mu$ pore 1-1 size filters and autoclaved $45 \mathrm{~min}$ at $121^{\circ}$. One carboy was fitted with a siphon to refill the feed carboy through a rubber covered port, connexion being made with a sterile syringe needle. Normally two 401 . carboys were sterilized per run. Weighed quantities of glycerol were also injected into the feed where required and its concentration calculated with reference to volume calibration marks inscribed on the carboy.

To start a run, shake-flask cultures were inoculated from stock agar slants and a small quantity of exponentially growing organisms introduced into the fermentor. Equipment set-up time was about 2 days; runs lasted 1 to 2 months. The system was generally trouble free; two of seventeen runs were interrupted for culture failure for some unknown reason and one for mechanical repairs.

An appropriate interval was allowed between adjustments to ensure that new steady-state values had been reached. Continuous recording of the population densities after a substrate concentration shift indicated a smooth asymptotic approach to the new steady-state value as predicted by the rate of change of substrate concentration. The composition of the medium in the fermentor will approach that of the feed medium in a mixed system such as this by a factor of $1-e^{-r \Theta}$ in $r$ hours and is $99 \%$ complete after 4.6 fermentor volumes have been pumped through. Continuous recording of population densities showed that new steady-state populations were approached in a smooth asymptotic manner. Thus 5-10 vol. were allowed to pass through the fermentor before taking steady-state readings, depending on the magnitude of the change in population density. When the composition of the sparged gas into the feed was changed resulting population densities showed that equilibration was essentially complete in $24 \mathrm{hr}$. Here a minimum of 2 days was allowed between steady-state readings.

After changing the dilution rate, assuming no lag in the biological response of the system, the approach to new steady-state values should be about $99 \%$ complete after dilution by only one fermentor volume at the new rate. For this adjustment at least five fermentor volumes were allowed to pass through for conditions to stabilize.

Population density measurements were made primarily with an electronic counter 
calibrated against direct dry-weight determinations; optical extinction measurements and occasional plate counts were also made. The cell suspension was brought to a suitable conductivity with sodium chloride $7.5 \mathrm{~g}$./1. and sodium sulphate $2.5 \mathrm{~g} . /$ l., the latter being added to remove traces of dissolved oxygen and to stop growth of the oxygen-limited culture during the analysis period. Samples were diluted to 60,000 organisms $/ \mathrm{ml}$. or less to decrease coincidence errors where necessary. A $50 \mu$ orifice permitted good electronic sizing. Little orifice fouling was encountered during counts since the media and diluent had been pre-filtered. Oscilloscope pulse height was taken as proportional to individual cell volumes (Kubitscheck, 1956).

Dry-weight measurements were made with a Cahn electronic microbalance. The pans were enclosed in a tube wound with heating coils, which could be evacuated for rapid drying. Samples of organisms were collected on tared $13 \mathrm{~mm}$. diam. $0.45 \mu$ pore size filters, washed and then dried in place on the balance pan. About $1 \mathrm{mg}$. organisms was collected on a $7 \mathrm{mg}$. filter. Accuracy was limited by the facility with which the filter disc was removed from its holder on humid days. Static electricity made weighing impossible on dry days. Triplicate sample weight variation seldom exceeded $5 \%$. Samples were removed for analysis through a syringe needle extending into the fermentor and capped with a small cork.

\section{RESULTS AND DISCUSSION}

Population density data were collected at a series of growth rates and feed oxygen values to determine fermentor oxygen concentrations according to equation (7). The maximum growth rate was established in batch culture at $0.506 \mathrm{hr}^{-1}$; this value of $\mu_{\max }$. persisted at the low populations near that of operation. Most data were

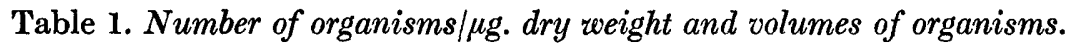

$\begin{array}{cccc}\text { Growth rate } & \begin{array}{c}\text { No. organisms } / \mu \mathrm{g} . \\ \text { dry wt. } \\ \left(\% \mu_{\text {max. }}\right)\end{array} & \begin{array}{c}\text { Average } \\ \text { volume/organism }\end{array} & \begin{array}{c}\text { Most frequent } \\ \text { volume/organism } \\ \left(\times 10^{5}\right)\end{array} \\ 30 & 1.71 & \left(\mu^{3}\right) & \\ 50 & 1 \cdot 04 & 41 & 34 \\ 80 & 0.83 & 48 & 37 \\ & & 60 & 43\end{array}$

collected at 30,50 and $80 \%$ of this growth rate. The sizes of the organisms were significantly larger at faster growth rates as shown in Table 1. These data were taken from size distribution curves of the type shown in Fig. 3 and in conjunction with dryweight measurements. Variation in extinction at $625 \mathrm{~m} \mu /$ unit mass of organism was more than twofold over the growth rates observed. The yield of organism/g. oxygen utilized decreased from $1.5 \mathrm{~g}$. organism/g. oxygen near $\mu_{\max }$, down to a yield constant of about 1.0 at $5 \%$ of $\mu_{\max }$ as shown in Fig. 4. These data were taken directly from dry-weight measurements at various growth rates with limiting oxygen at $31.5 \mathrm{mg} . / 1$. Oxygen utilized was taken as input of dissolved oxygen less a small correction for unused oxygen escaping in the effluent. This correction was made according to the Michaelis-Menten equation, $\mu=\mu_{\max } S /\left(K_{s}+S\right)$ and increased with growth rate. The value of $K_{s}$ for oxygen was taken as $0.45 \mathrm{mg} . / 1$. as determined below. The growth rate was taken as the dilution rate of the continuous culture system. 
Fermentor oxygen concentrations at the three growth rates were determined by extrapolation of steady-state population values to zero population as shown in Figs. 5-7. Here population measurements were based on numbers of organisms since the experimental and statistical problems involved in obtaining size distribution data at the very small population densities used prevented accurate cell volume determinations. These small population densities were also below the lower limits for reasonable accuracy of the dry-weight measurements. While extinction measurements were obtained, they were not of a high order of accuracy at the low population densities used.



Fig. 2

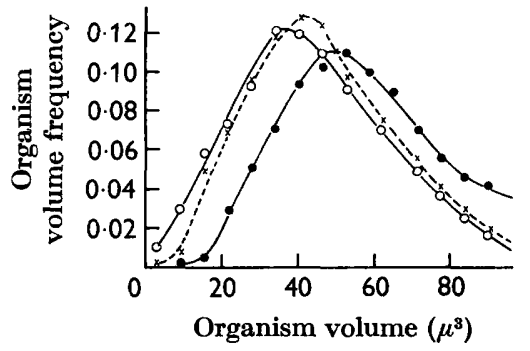

Fig. 3

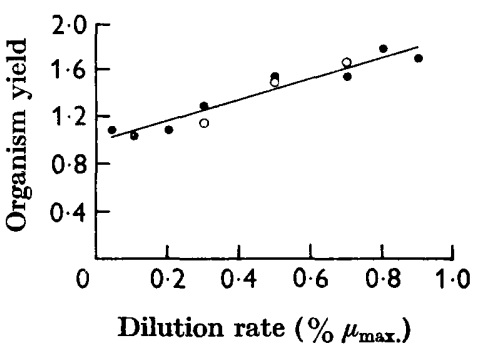

Fig. 4

Fig. 2. Steady-state population density against glycerol concentration. The growth rate is half maximum or $0 \cdot 253 \mathrm{hr}^{-1}$. Feed dissolved oxygen is held constant at $6 \cdot 2 \mathrm{mg} . / 1$.

Fig. 3. Volumes of organisms distribution at three growth rates limited by dissolved oxygen concentration. Growth rates are: $O, 30 \% ; \times, 50 \%$ and $0,80 \%$ maximum. Curves are reduced to a common total organism volume.

Fig. 4. Steady-state yield of organism from oxygen plotted against dilution rate. organism dry wt. /mg. oxygen used. $O$, organism volume (arbitrary units)/mg. oxygen used.

Table $2 . \mathrm{O}_{2}$ concentration and yield constants at various growth rates.

$$
\begin{gathered}
\text { Growth rate } \\
\left(\% \mu_{\max .}\right)
\end{gathered}
$$

30

50

80

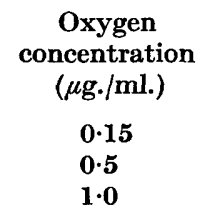

Yield
constant*

$1 \cdot 2$

1.5

$1 \cdot 8$
Yield constant†

$1 \cdot 2$

$1 \cdot 4$

$1 \cdot 7$

* Yield of organism from direct dry-weight measurements (Fig. 4).

$\dagger$ Yield of organism calculated from slope of $X=\gamma\left(S_{0}-S\right)$ plot (Figs. 5-7).

The fermentor oxygen concentrations obtained from Figs. 5 -7 and summarized in Table 2 were computer fitted to the hyperbola described by the MichaelisMenten equation to obtain the concentration of oxygen at which growth proceeded at half its maximum rate. Values for extracellular oxygen concentrations obtained in a similar manner by using extinction values and plate count data were approximately the same as those shown in Table 2. The relationship between growth velocity and oxygen concentration taken from the electronic counter data is shown in Fig. 8. The curve drawn is the best hyperbola fitting these data. Deviation of the data from the curve drawn is within experimental error. The Michaelis constant for oxygen-limited growth of this yeast was thus determined to be $0.61 \mathrm{mg}$./1. with a standard error of $0.4 \mathrm{mg} . / \mathrm{l}$. 
Several assumptions were made to determine that an oxygen concentration of $1 \cdot 4 \times 10^{-5} \mathrm{M}$ will allow this yeast to grow at half its maximum rate. Growth rate must be only a function of limiting nutrient concentration. Where this is true a plot of input substrate concentration against population density at a single through-put rate will be linear, as shown in Fig. 9. A curve toward the substrate axis which has

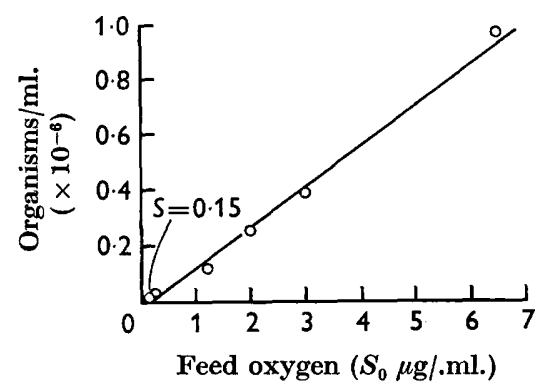

Fig. 5

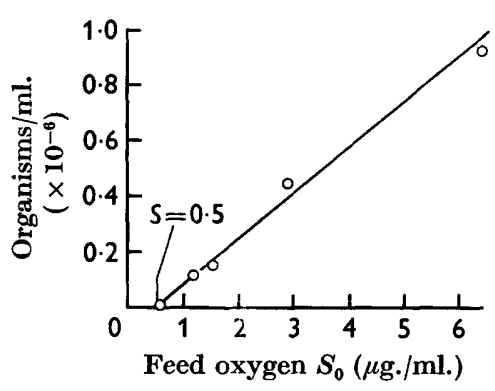

Fig 6

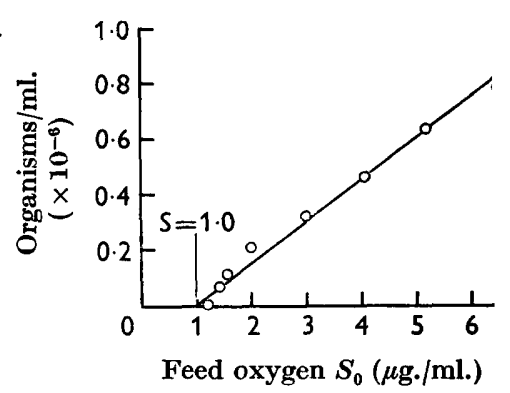

Fig. 7

Fig. 5. Steady-state population densities plotted against oxygen concentration in the feed at $30 \%$ of the maximum rate. The line extrapolates to an extracellular oxygen concentration of $0.15 \mathrm{mg}$. $/ \mathrm{l}$. at this growth rate.

Fig. 6. Same as Fig. 5 except growth rate is $\mathbf{5 0} \%$ maximum.

Fig. 7. Same as Fig. 5 except growth rate is $80 \%$ maximum.

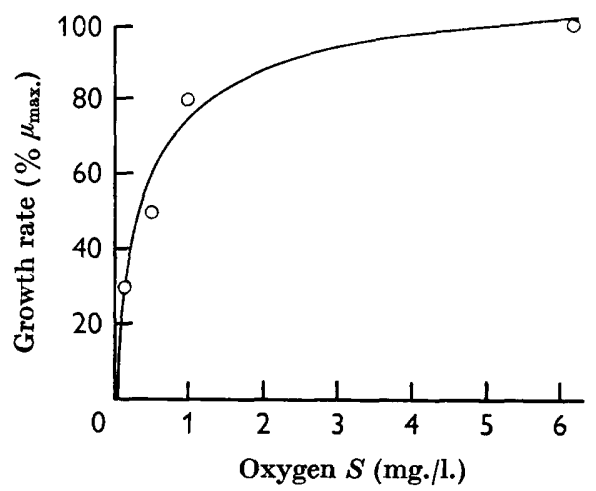

Fig. 8

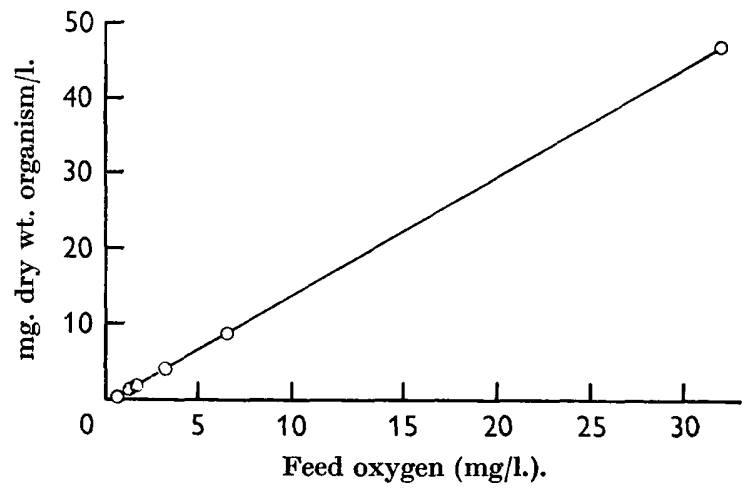

Fig. 9

Fig. 8. Growth rate of Torulopsis utilis plotted against extracellular dissolved oxygen concentration.

Fig. 9. Steady state-organism mass plotted against dissolved oxygen concentration in the feed at $50 \%$ of the maximum growth rate.

sometimes been reported probably represents partial limitation by other constituents of the medium or an effect of population density on the yield of organisms from substrate. Such a curve resulted in the present system when the carbon source was glucose and facultatively anaerobic metabolism could occur. Glycerol can only be used aerobically, however, and there was no effect of this carbon source on oxygen-limited population density when the glycerol concentration was varied over a 100-fold range. This is shown by the sharp break in Fig. 2. Oxygen concentration was the only factor which controlled both growth rate and population density. Change in the growth rate did, however, affect the size of the organisms, as 
shown in Fig. 3. Extrapolation to a negative substrate concentration in this plot as sometimes found represents a background of limiting substrate in the feed.

The second assumption requiring steady-state operation was not established or closely approximated until operation was shifted to yeast rather than bacteria eliminating the problem of adhesion to the reactor wall. In a larger fermentor, or when working with higher population densities, this phenomenon might well have been overlooked. However, here the effect on resulting data was large; the consistency of the data over extended periods of operation at small population densities substantiates this assumption.

The third requirement is for perfect mixing, which implies a homogeneous dispersion of substrate as well as organisms. Gross mixing in this small fermentor appeared to be better than in larger conventional fermentors as judged from the observation of injected dyes. Improved mixing at the surface of the organisms would probably not affect the data since the diffusivity of oxygen through water is such that a stagnant film of medium a few microns thick around the organisms should have little effect on the rate of growth.

The last assumption, that all the organisms are viable, must hold for the dilution rate to be equal to the growth rate. Steady state could theoretically be achieved with a proportion of the organisms produced being non-viable. In this case, the net growth rate, i.e. the growth rate minus the death rate, is equal to the dilution rate. In these experiments where kinetic data were gathered, i.e. at dilution rates between 30 and $80 \%$ maximum, plate counts and electronic counter data were the same within experimental error. Thus, essentially all of the organisms were viable at these high growth rates. However, as the dilution rate was decreased below $30 \%$ of the maximum the resulting yield data can be interpreted to suggest the production of increasingly large numbers of metabolically inactive organisms. It can be seen from Fig. 4 that increasingly lower yields were associated with lower growth rates. If it be assumed that the culture utilizes a portion of the oxygen for endogenous metabolism at a constant rate dependent only on the mass of organisms, and the rest for growth processes at the associated rate, an endogenous metabolism rate constant can be determined. At growth rates above $30 \%$ maximum where yield data are of greatest precision, a plot of $1 / \mu$ versus $1 / \gamma$ gives a straight line as predicted from the model with an endogenous metabolism rate constant of $0.057 \mathrm{~g}$. oxygen consumed $/ \mathrm{g}$. organism/hr. Marr (1963) determined a similar rate constant, $0.028 \mathrm{hr}^{-1}$, for Escherichia coli with glucose as the limiting nutrient. The yield data presented conform to the above model at lower growth rates only when it is assumed that increaxingly large proportions of the organisms are dead. This assumption predicts that $79 \%$ of the culture would be dead at the lowest growth shown. While the data are not sufficient to present a complete explanation, they clearly suggest matters to be given close attention when working at low growth rates.

The $K_{s}$ value of $1 \cdot 4 \times 10^{-5} \mathrm{M}$ for oxygen is significantly higher than the range of values Longmuir (1953) obtained for several yeast, bacterial, and subcellular preparations; $6.45 \times 10^{-7} \mathrm{M}$ for Baker's yeast, $2.22 \times 10^{-8} \mathrm{M}$ for Escherichia coli. The data of Longmuir were obtained by measuring polarographically the short-term rate of oxygen removed by an oxygen-starved suspension of organisms. It seems likely that this procedure measures a different phenomenon than the $K_{s}$ value for growth reported here. The $K_{s}$ for growth probably measures the rate of some rate- 
limiting reaction within the organism which is regulated, perhaps indirectly, by oxygen concentration. Short-term oxygen uptake, however, probably represents the ability of oxygen-starved organisms to absorb nutrient. This is supported by the direct correlation which Longmuir found between $\boldsymbol{K}_{\boldsymbol{s}}$ for oxygen and size of organism. The limited data available here for Torulopsis utilis show no such correlation between $\boldsymbol{K}_{s}$ values for growth and size of organism.

The values of $1.4 \times 10^{-5} \mathrm{M}$ for oxygen and $4.9 \times 10^{-5} \mathrm{M}$ for glycerol are similar to values obtained for growth by other workers. Monod (1942) reported $2.2 \times 10^{-5} \mathrm{M}$ for glucose with Escherichia coli in batch culture. Rao (1962) reported $2.1 \times 10^{-5} \mathrm{M}$ when using the experimental approach used here with $E$. coli. The direct analytical data of Davies et al. (1962) who used a haemolytic streptococcus and growth limited by various amino acids indicated $K_{s}$ values from $10^{-3}$ to $10^{-5} \mathrm{M}$. Extrapolation of their data obtained at various feed amino acid concentrations according to the method presented here yields values that agree well with their own analytical values.

While data given here are not of sufficient precision to make statements about the exact shape of the oxygen-limited growth velocity curve with Torulopsis utilis, they do indicate the concentration range of dissolved oxygen at which aerobic growth proceeds at a reasonable rate. The consistency of the data demonstrate the advantage of obtaining growth information from steady-state systems.

\section{REFERENCES}

Button, D. K. (1964). Continuous culture of Torulopsis utilis with oxygen the limiting nutrient. Ph.D. thesis, University of Wisconsin.

Danckwerts, P. V. (1954). Continuous flow of materials through processing units. Industr. Chem. Mfr. 30, 102.

Davies, H. C., Karush, F. \& Rudd, J. H. (1965). Effect of amino acids on steady state growth of group A hemolytic streptococcus. J. Bact. 89, 421.

Herbert, D., Elsworth, R. \& Teling, R. C. (1956). The continuous culture of bacteria; a theoretical and experimental study. J. gen. Microbiol. 14, 601.

KuBITSCHECK, H. E. (1958). Electronic counting and sizing of bacteria. Nature, Lond. 182, 234.

LoNGMUIR, I. S. (1954). Respiration rate of bacteria as a function of oxygen concentration. Biochem. J. 57, 81.

MARR, A. G., Nilson, E. H. \& Clark, D. J. (1963). The maintenance requirement of Escherichia coli. Ann. N.Y. Acad. Sci. 102, 536.

Monod, J. (1942). Recherches sur la Croissance des Cultures Bactériennes. Paris: Hermann.

Monod, J. (1950). La technique de culture continue: théorie et applications. Ann. Inst. Pasteur 79, 890.

RAo, K. S. (1962). The kinetics of continuous culture of Escherichia coli. M.S. Thesis, University of Wisconsin. 